\title{
Akupunktur und Moxibustion bei Pneumonie durch Infektion mit dem Corona-Virus (SARS-CoV-2)
}

\author{
Liang Fanrong, Chengdu \\ Übersetzt von Gerlinde Wislsperger, München
}

Zusammenfassung: Der Autor beschreibt die Möglichkeiten der Aku-Moxi-Therapie bei Lungenentzündung im Gefolge einer Infektion mit dem SARS-CoV-2-Virus, wie sie in offiziellen Gremien in der VR China von ausgewählten Experten als Anleitung für das Krankenhausfachpersonal zusammengestellt wurde.

Am Anfang des Artikels macht er mit einigen historischen Zitaten deutlich, dass sich die Chinesische Medizin seit langem mit epidemischen Krankheiten beschäftigt und Behandlungsmöglichkeiten dagegen bereithält.
Für seine Therapie-Anleitung differenziert er drei Stadien der Krankheit: die Verdachts-, die Krankheits- und die Rekonvaleszenzphase; bei der Beschreibung der Interventionen stehen Akupunktur, Moxibustion und Ohrakupunktur im Vordergrund. Neben dem hauptsächlichen Therapieprinzip, die Abwehrkräfte zu stärken, werden jeweils auch Punkte für Begleiterscheinungen wie verschiedene Formen des Hustens, Fieber, Kraftlosigkeit oder Übelkeit und Erbrechen aufgeführt. Daneben werden Akupressur, Tuina und Qigong/Taiji als Möglichkeiten genannt, mit denen Patienten selbst aktiv an der Vorbeugung und Therapie mitwirken können.

Schlüsselwörter: Pneumonie · SARS-CoV-2 - Epidemische Erkrankungen · Kräftigung der Abwehrkräfte · Aku-Moxi-Therapie · Ohrakupunktur · Tuina · Akupressur · Übungen zum Leiten und Dehnen (daoyin) · Qigong · Taiji

Chin Med 2020;35:79-86

\section{Acupuncture and moxibustion to treat pneumonia arising from Coronavirus (SARS-CoV-2) infection}

Abstract: The author describes the ways in which acu-moxi therapy can be used to treat pneumonia following infection with the SARS-CoV-2-Virus, as proposed by selected experts in official bodies in the People's Republic of China as guidance for hospital staff.

At the beginning of the article, he makes it clear with a few historical quotations that Chinese medicine has been dealing with epidemic diseases for a long time and offers a range of treatment options for combating them.
For the purpose of therapy, he differentiates between three stages of the disease: the suspected, the disease and the convalescence phase. In describing the interventions, acupuncture, moxibustion and ear acupuncture are of primary importance. In addition to focusing on the main therapeutic principle of strengthening the immune system, the article also mentions points for treating accompanying symptoms such as various forms of coughing, fever, weakness or nausea and vomiting. The possibilities of acupressure, Tuina and Qigong/ Taiji are also considered as ways in which patients can actively participate in prevention and therapy.

Key Words: Pneumonia · SARS-CoV-2 · Epidemic diseases · Strengthening the immune system · Acu-moxi therapy · Ear acupuncture - Tuina - acupressure - Exercises to guide the qi and stretch the body (daoyin) - Qigong · Taiji 
Historische Aussagen zu epidemischen Erkrankungen

Einsatz der Aku-Moxi-Therapie bei Prävention und Therapie

Zitate aus klassischen Werken zu epidemischen Erkrankungen
Bei der SARS-CoV-2-Pneumonie handelt es sich um eine akute Infektionskrankheit der Atemwege, die allgemein hoch ansteckend ist und von der daher für die Sicherheit und Gesundheit der Bevölkerung eine ernsthafte Gefährdung ausgeht.

Die neue Coronavirus-Pneumonie ist aus Sicht der Chinesischen Medizin den „epidemischen Krankheiten“(疫病 yibing) zuzurechnen. Die Chinesische Medizin hat über Jahrtausende eine Fülle von Erfahrungen bei der Behandlung von Epidemien gesammelt. Aku-Moxi-Therapie ist ein wichtiger Bestandteil der Traditionellen Chinesischen Medizin, mit ihren charakteristischen Besonderheiten und Vorzügen hat sie in der Geschichte des Kampfes gegen epidemische Krankheiten einen wesentlichen Beitrag geleistet. In den klassischen Werken zur Chinesischen Medizin findet man Aufzeichnungen zur Prävention und Behandlung von epidemischen Krankheiten mit Akupunktur und Moxibustion. Zum Beispiel hat der Tang-zeitliche Medizingelehrte und Arzt Sun Simiao (581-682) in seinem Werk „Wichtige Rezepturen, die tausend Goldstücke wert sind" (Qianjin yaofang, im 7. Jh. entstanden) vorgebracht:

„Beamte aus den Staaten Wu und Shu, die umherreisen, benötigen häufig Moxibustionen an drei oder zwei Stellen an ihrem Körper ... auf diese Weise können epidemische Erkrankungen, Wechselfieber aufgrund ,Wärme' und toxisches Qi nicht auftreten."

Der Arzt Li Shizhen (1518-1593) aus der Ming-Zeit erklärte in seiner "Systematischen Drogenkunde“ (Bencao gangmu, um 1596 fertiggestellt): „Artemisiae argyi folium (Aiye) ... wenn man (dieses Kraut) abbrennt, durchdringt man alle Leitbahnen und kann hundert Arten von krankmachenden Einflüssen behandeln. Auf diese Weise kann man Menschen mit schweren Krankheiten zu Gesundheit verhelfen, und ihre Wirkung ist stark."

Derlei Aufzeichnungen machen deutlich, dass man mit der Aku-MoxiTherapie ansteckenden Krankheiten vorbeugen und sie wirksam behandeln kann. Die gegenwärtige klinische und experimentelle Forschung zeigt, dass sich mithilfe der Aku-Moxi-Therapie die Abwehrkräfte des Menschen regulieren lassen, dass sie entzündungs- und infektionshemmende Wirkungen hat und bei ansteckenden Krankheiten eine relativ gute präventive und therapeutische Wirkung entfalten kann. Auch bei der Prävention und Therapie der plötzlich aufgetretenen neuen Pneumonie durch SARS-CoV-2 hat der Einsatz der traditionellen chinesischen Akupunktur gute Ergebnisse erbracht.

Nachdem wir unser Verständnis dieser neuartigen Pneumonie vertiefen und Erfahrungen bei der Diagnose und Behandlung mit Akupunktur sammeln konnten, haben wir gemäß den offiziellen nationalen Richtlinien einen Kreis von Experten zusammengestellt und in diesem Rahmen die „Richtlinien zur Akupunkturintervention bei SARS-CoV-2-Pneumonie (2. Auflage) “ formuliert, auf die sich medizinische Fachkräfte bei der Durchführung der Akupunktur beziehen und durch die sie bei der Behandlung ambulanter Patienten angeleitet werden sollen.

\section{Prinzipien der Intervention mit Akupunktur und Moxibustion}

Während der Epidemie sollten Interventionen mit Akupunktur und Moxibustion in die Gesamtsituation gemäß den nationalen Leitlinien integriert werden. Gleichzeitig sollten die Besonderheiten der Aku-Moxi-Therapie umfassend bedacht werden, so dass sie gezielter wirken kann.

SARS-CoV-2-Pneumonie ist eine der für gewöhnlich sehr ansteckenden „fünf epidemischen Krankheiten (五疫 wuyi), die sich rasch ausbreiten und deren 
Symptome unabhängig vom Alter (des Patienten) ähnlich sind“ (72. Kapitel der „Unbefangenen Fragen“ im „Inneren Klassiker des Gelben Fürsten“, Huangdi Neijing Suwen, etwa 1. Jh. v.u.Z., im 8. und 11. Jh. überarbeitet). Wenn "Epidemisches“(疫戻 yili) über Mund und Nase eindringt, greift es meistens zuerst die dem yin maior (Großes Yin, taiyin) zugeordneten oo. pulmonalis et lienalis (Fk „Lunge" und „Milz", fei pi) an und affiziert auch die dem splendor yang (Überstrahlung des Yang, yangming) zugeordneten oo. stomachi et intestini crassi (Fk „Magen“ und „Dickdarm“, wei dachang), wobei die Krankheitszeichen relativ mild sind. Bei einer geringen Anzahl Infizierter werden die oo. pericardialis, hepaticus et renalis (Fk „Herzbeutel“, „Leber“ und „Niere“, xinbao gan shen) kontravektiv (gegenläufig, $n i$ ) angegriffen, wodurch Yin und Yang sich voneinander trennen, so dass diese Patienten schwer erkranken. Die Krankheit verändert sich rasch, sie besitzt einen klar erkennbaren, zentralen Pathomechanismus und einen sich gesetzmäßig entwickelnden Verlauf der Krankheitszeichen.

Nach dem Grundsatz, dass „die Leitbahnen sich innen mit den Funktionskreisen verbinden und sich außen mit den Gliedmaßen vernetzen“, kann man Foramina an den Gliedmaßen akupunktieren, um die sinarteriae cardinales et reticulares (Haupt- und Netzleitbahnen, jingluo) durchlässig zu machen, Qi und Xue zu regulieren und zu harmonisieren und so die Abwehrkraft des Körpers zu stärken. Auf diese Weise lässt sich die eingedrungene „epidemische Heteropathie (Schrägläufigkeit, xie) mit Schmutzig-Trübem" beseitigen und anschließend die Orthopathie (Geradläufigkeit, zheng) stärken. Gleichzeitig kann man das qi cardinale (Leitbahn-Qi, jingqi) stimulieren, die Selbstschutzfähigkeit der oo. horreales et aulici (Speicher- und Durchgangsfunktionskreise, zangfu) erhöhen und die Schädigung der oo. horreales (Speicherfunktionskreise, zang) durch das „Epidemisch-Toxische“ (yidu) reduzieren.

Beim Einsatz der Aku-Moxi-Therapie wird nach drei Phasen differenziert: Sie kann in der Phase bei Verdacht auf eine Infektion mit SARS-CoV-2, in der Phase der gesicherten Diagnose und in der Genesungsphase erfolgen. Als Hauptforamina werden Punkte gewählt, die auf die Therapie des zentralen Pathomechanismus gerichtet sind, und diese sind um weitere Punkte zu ergänzen, mit denen zusätzliche klinische Krankheitszeichen behandelt werden können. Dabei sollte das Prinzip, „wenige und essentielle Foramina auszuwählen“, beachtet werden.

Bei Patienten in der Rekonvaleszenzphase sollte eine rehabilitative Aku-MoxiTherapie eine zentrale Funktion übernehmen. Dazu wird empfohlen, neue akupunkturbasierte Rehabilitationsabteilungen für SARS-CoV-2-Pneumonie einzurichten.

Patienten sollten zudem dazu ermutigt werden, unter Anleitung von Akupunkturexperten eigenhändig oder mit Hilfsmitteln Moxibustion, Aufkleben von Pflastern auf Foramina, Akupressur etc. durchzuführen, um die Therapie zu unterstützen und eine körperliche und seelische Genesung zu erreichen.

\section{Methoden der Intervention mit Akupunktur und Moxibustion}

\section{1) Aku-Moxi-Therapie während des Verdachts auf eine Infektion mit SARS-CoV-2}

Mit dem Behandlungsziel, das orthopathische Qi (geradläufiges Qi, zhengqi) anzuregen und die organische Immunabwehr zu erhöhen, wählt man als Hauptforamina IC11/Di11 („Gekrümmter Teich“, quchi), S36/Ma36 („Dritter Weiler am
Pathomechanismus und Therapieprinzip

Differenzierung von drei Stadien der Erkrankung

Möglichkeiten der unterstützenden Selbstbehandlung mit Moxibustion, Akupressur

Foramina zur Therapie der Kernerkrankung und von Begleiterscheinungen bei Verdacht auf eine Infektion 
Technische Anleitung bei der Akupunktur und Moxibustion

Anwendung von Tuina und Akupressur in der Beobachtungsphase
Fuß“, zusanli), F20/Gb20 („Teich des Windes“, fengchi), V13/Bl13 („Einflusspunkt des ,Lungen'-Funktionskreises“, feishu) und Rs6/Ren6 („Meer des Qi“, qihai).

Bei Patienten, die begleitend unter Fieber, Trockenheit des Halses und trockenem Husten leiden, sollte man zusätzlich auf den Foramina Rg14/Du14 („Punkt aller Strapazen“, dazhui), Rs22/Ren22 („Bresche des Himmels“, tiantu) und P6/Lu6 („Die Tiefe des Lochs“, kongzui) behandeln. Bei Patienten, die begleitend unter Übelkeit und Erbrechen und breiigen Stühlen leiden und eine gedunsene Zunge mit einem klebrigen Zungenbelag und pp. lenes („nachgiebige“ Pulse, mairu) haben, ergänzt man die Punkte Rs12/Ren12 („Sammlungspunkt des ,Magen'-Funktionskreises“, zhongwan), S25/Ma25 („Angel des Himmels“, tianshu) und Pc6/Pe6 („Inneres Passtor“, neiguan). Bestehen begleitend Müdigkeit, Kraftlosigkeit und Appetitmangel, fügt man die Punkte Rs12/Ren12 („Sammlungspunkt des ,Magen'-Funktionskreises“, zhongwan) und Rs4/Ren4 („Das erste der Passtore", guanyuan) hinzu. Wenn gleichzeitig klares Nasensekret fließt, ziehende Schmerzen in Schultern und Rücken bestehen, die Zunge blass und weiß belegt ist und die Pulse languidi („,behäbig“, huan) sind, sollte man die Foramina S25/Ma25 („Angel des Himmels“, tianshu), V12/Bl12 („Pforte der Winde“, fengmen) und Rg14/Du14 („Punkt aller Strapazen“, dazhui) ergänzen.

\section{Vorgehensweise bei der Aku-Moxi-Therapie}

Für gewöhnlich werden Akupunktur und Moxibustion in Kombination eingesetzt, wobei die Moxibustion im Vordergrund steht. Die Akupunktur erfolgt routinemäßig; nach dem Einstechen der Nadel und Erreichen des Qi führt man das „ausgewogene Suppletieren und Dispulsieren“ (平补平泻 pingbu pingxie) durch, wobei die Nadel bei jedem Foramen 20 bis 30 Minuten liegen gelassen werden sollte. Während dieser Zeit kann man hauptsächlich die Foramina S36/ Ma36 („Dritter Weiler am Fuß“, zusanli) beidseitig, Rs6/Ren6 („Meer des Qi“, qihai), Rg14/Du14 („Punkt aller Strapazen“, dazhui) und S25/Ma25 („Angel des Himmels", tianshu) beidseitig mit erwärmter Nadel oder mit einer Moxazigarre moxen. Falls die Möglichkeiten begrenzt sind, ist es möglich, nur eine der beiden Methoden einzeln einzusetzen. Die Therapie sollte einmal täglich stattfinden.

\section{Pressen auf Akupunkturpunkten (Akupressur) und Leitbahnabschnitten}

Die Akupressur kann auf den oben genannten Haupt- und Zusatzforamina ausgeführt werden. Dabei sollte jeder Punkt etwa eine Minute lang mit mittelstarker Kraft abwechselnd geknetet ${ }^{1}$ und gepresst (按揉 anrou) werden. Für das Pressen auf Leitbahnabschnitten kann man

- die cardinalis pulmonalis yin maioris manus (Hauptleitbahn des Fk „Lunge“ Großes Yin der Hand, shou taiyin fei jing) vom Foramen P5/Lu5 („Moorsee am Fußpunkt“, chize) bis P9/Lu9 („Großer Wasserschlund“, taiyuan) oder

- die cardinalis intestini crassi splendoris yang manus (Hauptleitbahn des Fk „Dickdarm“ Überstrahlung des Yang der Hand, shou yangming dachang jing) von IC11/Di11 („Gekrümmter Teich“, quchi) bis IC5/Di5 („Yang-Bach“, yangxi) oder

\footnotetext{
Beim Kneten (揉 rou) handelt es sich um eine Massagetechnik aus der Tuina-Massage, die hier mit dem Daumen ausgeführt wird. Sie besteht in einer langsamen, gleichmäßig kreisenden Bewegung auf einem Hautareal, das während der Behandlung nicht verlassen wird (vgl. Han Chaling, Leitfaden Tuina, Elsevier 2013, S. 95ff).
} 
- die cardinalis stomachi splendoris yang pedis (Hauptleitbahn des Fk „Magen“ Überstrahlung des Yang der Hand, zu yangming wei jing) von S36/Ma36 („Dritter Weiler am Fuß“, zusanli) bis S41/Ma41 („Befreiter Wasserlauf“, jiexi) oder

- die cardinalis lienalis yin maioris pedis (Hauptleitbahn des Fk „Milz“ Großes Yin des Fußes, $z u$ taiyin pi jing) von L9/Mi9 („Die Quelle am Yin-Grabhügel“, yinlingquan) bis L6/Mi6 („Die Verbindung der drei Yin“, sanyinjiao) wählen.

Zur Behandlung reibt der Therapeut mit dem Daumenballen einer Handfläche gerade, rasch und kräftig von proximal nach distal und zurück direkt auf der Haut (oder auf einem dünnen Baumwollstoff über der Haut). Diesen Behandlungsschritt wiederholt man 100- bis 120-mal und behandelt auf diese Weise jeden der genannten Leitbahnabschnitte etwa eine Minute lang. Man kann auch die Technik des Klatschens (拍打 paida) ${ }^{2}$ einsetzen und je eine Minute auf jedem der Leitbahnabschnitte hin- und zurückbehandeln. Auch eine Selbstbehandlung der Patienten unter Anleitung des Arztes ist möglich.

\section{Ohrakupunktur}

Als Hauptpunkte wählt man Milz (98) ${ }^{3}$, Magen (87), Lunge (101), Nebennieren (13) und Endokrinium (22), bei Patienten, die begleitend unter Husten, Halsschmerzen und Fieber leiden, behandelt man zusätzlich auf den Ohrpunkten Ohrspitze (78), Hals (41) und Trachea (103); bei Patienten mit Magenschmerzen, Übelkeit, Erbrechen und breiigen Stühlen setzt man Dickdarm, Dünndarm (89) und Sanjiao (104) ein, und bei Patienten, die begleitend unter laufender Nase mit klarem Nasensekret, ziehenden Schmerzen an Schultern und Rücken klagen, ergänzt man die Ohrpunkte Äußere Nase (14) und Schulter (65). Bei Kopfschmerzen behandelt man zusätzlich die Punkte Stirn (33) und Kopfscheitel (36), bei Schlaflosigkeit Shenmen (55), Herz (100), Niere (95) und vorderes Ohrläppchen und bei Unruhe und Besorgtheit Shenmen (55), Herz (100) und Leber (76, 77, 97). Als Therapiemethode verwendet man Kügelchen oder Dauernadeln für die Ohrakupressur; das Ohr sollte gründlich desinfiziert werden, bevor Vaccariae semen (Wangbuliuxing) oder sterile Einwegnadeln appliziert und mit einem Pflaster festgeklebt werden. Dann presst und knetet man mit mäßiger Kraft, bis sich ein warmes Gefühl im Ohr ausbreitet. Der Patient sollte angeleitet werden, 3- bis 5-mal täglich 1 bis 3 Minuten lang selbst zu drücken und dabei soviel Kraft einzusetzen, dass der Schmerz gerade erträglich ist. Bei Hautverletzungen, Hautallergien oder Narben im Ohrbereich sind diese Methoden nicht indiziert.

\section{Therapie mit Übungen zum Leiten und Dehnen (daoyin 导引)}

Hier sind die ersten drei sowie die siebte Form der "Acht-Brokat-Übungen" (八段锦 Baduan jin) zu empfehlen: „,mit beiden Händen den Himmel stützen, um die Drei Wärmebereiche zu regulieren“, „links und rechts den Bogen spannen, als wollte man auf einen Adler zielen“, „einen Arm heben, um die Funktionskreise

2 Das Klatschen (拍打 paida) wird mit der ganzen Hand kräftig und geräuschvoll ausgeführt, die Finger werden dabei ausgestreckt aneinandergelegt und leicht gewölbt; der Therapeut sollte darauf achten, dabei sein Handgelenk völlig zu entspannen (vgl. Han Chaling, Leitfaden Tuina, Elsevier 2013, S. 110).

3 Die Nummerierung der Ohrpunkte basiert auf der von Manfred Angermaier im Leitfaden Ohrakupunktur, München 2018.
Ohrakupunktur zur Therapie von Kernerkrankung und Begleiterscheinungen in der Beobachtungsphase

Empfehlenswerte Qigong-Übungen bei Verdacht auf eine Infektion 
Aku-Moxi-Therapie der Kernerkrankung und der Begleiterscheinungen in der Krankheitsphase

Zusätzlich einsetzbare Foramina
Milz-Magen aufeinander abzustimmen“ und „die Fäuste ballen und zornig schauen, um das Qi zu mehren“. Oder man wählt die ersten beiden Formen aus dem „Klassiker zur Wandlung von Muskeln und Sehnen“ (易筋经 Yijin jing). Man kann auch die gesamte Abfolge der „Acht-Brokat-Übungen“(八段锦 Baduan jin), der Übungen aus dem „Klassiker zur Wandlung von Muskeln und Sehnen“ (易筋经 Yijin jing) oder Taijiquan praktizieren.

\section{2) Aku-Moxi-Therapie bei gesicherter Diagnose}

Entsprechend dem therapeutischen Prinzip, das orthopathische Qi (geradläufiges Qi, zhengqi) zu unterstützen und „epidemische Heteropathien“ („epidemische Schrägläufigkeiten", yixie) zu eliminieren, sind als Hauptforamina IC11/Di11 („Gekrümmter Teich“, quchi), Rg14/Du14 („Punkt aller Strapazen“, dazhui), V13/ Bl13 („Einflusspunkt des ,Lungen'-Funktionskreises“, feishu), P1/Lu1 („Versammlungshalle der Mitte“, zhongfu), S36/Ma36 („Dritter Weiler am Fuß“, zusanli) und Rs4/Ren4 („Das erste der Passtore“, guanyuan) angezeigt.

Wenn Patienten unter Fieber leiden, das nicht zurückgeht, ergänzt man die Punkte T5/3E5 („Äußeres Passtor“, waiguan) und F20/Gb20 („Teich des Windes“, fengchi), bei Patienten mit Kurzatmigkeit und Druckgefühl im Brustbereich nadelt man zusätzlich Rs22/Ren22 („Bresche des Himmels“, tiantu) und Pc6/Pe6 („Inneres Passtor“, neiguan); bei Patienten mit schleimigem Auswurf und Husten ergänzt man P7/Lu7 („Reihe von Lücken“, lieque) und S40/Ma40 („Üppige Fülle“, fenglong). Bei Patienten mit Diarrhö sollten zusätzlich S25/Ma25 („Angel des Himmels“, tianshu) und S37/Ma37 („Obere Enge des weiten Feldes“, shangjuxu) zum Einsatz kommen, bei Patienten, die begleitend unter deutlichem Fieber, Husten mit gelbem Auswurf und Obstipation mit unangenehmer Stuhlentleerung leiden, sollte man die Foramina T6/3E6 („Der fliegende Tiger“, feihu, bzw. "Seitlicher Abzugsgraben“, zhigou), S25/Ma25 („Angel des Himmels“, tianshu) und Rs22/Ren22 („Bresche des Himmels“, tiantu) hinzufügen. Wenn bei den Patienten begleitend erhöhte subfebrile Temperaturen oder leichtes Fieber, Übelkeit und Erbrechen und breiige Stühle auftreten und die Zunge blass oder blassrot, der Zungenbelag weiß oder weiß und klebrig ist, wählt man zusätzlich die Punkte S25/Ma25 („Angel des Himmels“, tianshu), L14/Mi14 („Knoten im Abdomen“, fujie) und Pc6/Pe6 („Inneres Passtor“, neiguan).

\section{Vorgehensweise bei der Aku-Moxi-Therapie}

Akupunktur und Moxibustion werden in Kombination eingesetzt, wobei die Moxibustion im Vordergrund steht. Nach Einstich der Nadel und Erreichen des Qi führt man nach Standardroutine Akupunktur sowie das „ausgewogene Suppletieren und Dispulsieren“ (平补平泻 pingbu pingxie) durch, die Nadeln sollten an jedem Foramen 20 bis 30 Minuten verweilen. Währenddessen sollte man mit erwärmter Nadel oder mit einer Moxazigarre 10 bis 15 Minuten lang moxen. Es sollte einmal täglich eine Behandlung erfolgen.

\section{Pressen auf Akupunkturpunkten (Akupressur) und Leitbahnabschnitten}

Die Intervention kann nach den Empfehlungen für die Akupressur während des medizinischen Beobachtungszeitraums (Verdacht auf eine Infektion) wie oben beschrieben erfolgen. 


\section{Ohrakupunktur}

Als Hauptpunkte sind die Ohrpunkte Nervus sympathicus, Lunge (101), Milz (98), Magen (87), Dickdarm, Endokrinium (22) und Nebennieren (13) indiziert. Wenn ein Patient begleitend deutliches Fieber hat, sollten die Punkte Ohrspitze (78) und Sanjiao (104) hinzugefügt werden, und bei Husten ergänzt man die Punkte Trachea (103) und Beruhigung der Keuchatmung (平喘 pingchuan). Bei begleitenden Schlafstörungen behandelt man zusätzlich Shenmen (55), vorderes Ohrläppchen, Herz (100) und Niere (95); bei Unruhe und Besorgtheit fügt man die Punkte Shenmen (55), Herz $(100)$ und Leber $(76,77,97)$ hinzu. Für die Therapie können Kügelchen für die Akupressur auf Ohrpunkten oder Dauernadeln angebracht werden.

\section{3) Aku-Moxi-Therapie in der Phase der Rekonvaleszenz}

Nach dem Prinzip, verbliebenes Toxisches zu klären und zu beseitigen, das $q i$ primum (ursprüngliches Qi, yuanqi) wiederherzustellen und die Genesung der oo. pulmonalis et lienalis (Fk „Lunge“ und „Milz“, fei pi) zu unterstützen, sind als Hauptforamina Pc6/Pe6 („Inneres Passtor“, neiguan), S36/Ma36 („Dritter Weiler am Fuß“, zusanli), Rs12/Ren12 („Sammlungspunkt des, Magen'-Funktionskreises“, zhongwan), S25/Ma25 („Angel des Himmels“, tianshu) und Rs6/Ren6 („Meer des Qi“, qihai) zu wählen.

Zusätzlich wählt man bei depletio qi pulmonale et lienale (energetische Schwäche des Qi der Fk „Lunge“ und „Milz“, fei pi qi xu) die Punkte Rs17/Ren17 („Vorhof der Brust“, shanzhong/tanzhong), V13/Bl13 („Einflusspunkt des ,LungenFunktionskreises“, feishu), P1/Lu1 („Versammlungshalle der Mitte“, zhongfu), Rs4/ Ren4 („Das erste der Passtore“, guanyuan) und L9/Mi9 („Die Quelle am YinGrabhügel", yinlingquan) und bei einer Schwäche von Yin und Qi die Foramina Rs17/Ren17 („Vorhof der Brust“, shanzhong/tanzhong), Rs8/Ren8 („Mitte des Nabels“, shenque), Rs4/Ren4 („Das erste der Passtore“, guanyuan), R7/Ni7 („Der zurückfließende Strom“, fuliu) und R3/Ni3 („Mächtiger Wasserlauf“, taixi).

\section{Vorgehensweise bei der Aku-Moxi-Therapie}

Akupunktur und Moxibustion werden in Kombination eingesetzt, wobei die Moxibustion im Vordergrund steht. Nach dem Einstechen der Nadel und dem Erreichen des Qi führt man standardmäßige Akupunktur sowie das ,ausgewogene Suppletieren und Dispulsieren“ (平补平泻 pingbu pingxie) durch, wobei die Verweildauer der Nadel an jedem Punkt 20 bis 30 Minuten beträgt. Begleitend kann mit erwärmter Nadel oder mit einer Moxazigarre jeweils 10 bis 15 Minuten lang gemoxt werden. Falls die Möglichkeiten begrenzt sind, kann man die Methoden auch einzeln einsetzen. Es sollte täglich eine Therapiesitzung stattfinden.

\section{Pressen auf Akupunkturpunkten (Akupressur) und Leitbahnabschnitten}

Nach den Empfehlungen für die Intervention während des medizinischen Beobachtungszeitraums (Verdacht auf eine Infektion) kann man, wie oben beschrieben, das Pressen auf einzelnen Akupunkturpunkten oder auf Leitbahnabschnitten ausführen.

\section{Ohrakupunktur}

Als Hauptpunkte sind die Ohrpunkte Milz (98), Magen (87), Lunge (101), Nebennieren (13) und Endokrinium (22) zu wählen. Bei Patienten, die sich kraftlos
Ohrakupunktur der Kernerkrankung und der Begleiterscheinungen in der Krankheitsphase

Foramina zur Unterstützung der Genesung

Tuina und Akupressur und in der Phase der Rekonvaleszenz 
Qigong-Übungen in der Rekonvaleszenzphase fühlen, sollte man zusätzlich auf den Punkten Leber $(76,77,97)$, Niere (95) und Mund (84) behandeln, bei Unwohlsein im Magen-Darm-Bereich ergänzt man die Punkte Dickdarm und Abdomen (43). Bei Patienten, die unter Palpitationen und Schlafstörungen leiden, sind zusätzlich die Punkte Shenmen (55), vorderes Ohrläppchen, Herz (100) und Niere (95) angezeigt, bei Patienten mit Unruhe und Besorgtheit die Punkte Shenmen (55), Herz (100) und Leber (76, 77, 97). Für die Akupressur können auf den Ohrpunkten Kügelchen oder Dauernadeln befestigt werden.

\section{Therapie mit Übungen zum Leiten und Dehnen (daoyin 导引)}

Patienten sollten die „Acht-Brokat-Übungen“ (八段锦 Baduan jin) oder die Übungen aus dem „Klassiker zur Wandlung von Muskeln und Sehnen“(易筋经 Yijin jing) regelmäßig durchführen. Es können daraus auch einzelne Übungen und Formen ausgewählt und praktiziert werden, die der jeweiligen körperlichen Verfassung entsprechen und systematisch erlernt und geübt worden sind.

\section{Literatur}

Angermaier M. Leitfaden Ohrakupunktur (7. Auflage). München: Elsevier, 2018. Engelhardt U, Hildenbrand G, Zumfelde-Hüneburg C (Hrsg.). Leitfaden Qigong (2. Auflage). München: Urban \& Fischer in Elsevier; 2014.

Han Chaling: Leitfaden Tuina (3. Auflage). München: Urban \& Fischer in Elsevier, 2013. Hempen C-H: dtv-Atlas zur Akupunktur (10. Auflage). München: dtv, 2011.

\section{Zum Autor}

Prof. Liang Fanrong hat seit 1974 sein Leben der Akupunktur und Moxibustion gewidmet. Er war bis Anfang 2017 Präsident der TCM-Universität Chengdu und ist Vize-Präsident des Chinesischen Akupunktur-Ärzte-Verbandes. Er ist ein weltweit renommierter Wissenschaftler und Forscher und hat als Arzt eine reiche Erfahrung bei der Behandlung neurologischer, gynäkologischer und gastrointestinaler Krankheiten. Aktuell leitet er den Forschungsschwerpunkt zur Akupunkturpunkt-Spezifität. Seine Arbeiten wurden bereits vielfach ausgezeichnet und auch im Westen hochrangig publiziert( wie z.B. Liang F et al. Acupuncture as Adjunctive Therapy for Chronic Stable Angina. A Randomized Clinical Trial. JAMA Internal Medicine 2019. https://doi.org/10.1001/jamainternmed.2019.2407)

\author{
Korrespondenzadresse \\ Prof. Liang Fanrong \\ Vice-President of Chengdu TCM University, Dean of Graduate \\ School of Chengdu TCM University \\ 37 Shi-er-qiao Rd. \\ Chengdu Sichuan 610075 China \\ E-Mail:LFR@cdutcm.cn
}

\section{Einhaltung ethischer Richtlinien}

Interessenkonflikt: Liang Fanrong gibt an, dass kein Interessenkonflikt besteht. Dieser Beitrag beinhaltet keine Studien an Menschen oder Tieren. 


\section{Kommentar}

Von Dr. Josef Hummelsberger, München

\section{Akupunktur und Moxibustion bei COVID-19}

Basierend auf den Grundlagen der Chinesischen Medizin, Vorerfahrungen und Studien aus der SARSEpidemie 2003 und der Influenzawellen mit H1N1 in den letzten Jahren (Quellen: Zishan Gao: Translation of the Guidelines 6th edition National Health Commission of the PR China, Nanjing March 2020) wurden auch Vorschläge zu Akupunktur und Moxibustion zur Stützung des Immunsystems und Behandlung von leichten bis mittelschweren COVID-19-Patienten diskutiert und erarbeitet, die wir hier als Ergänzung zum Beitrag von Professor Liang Fanrong kurz zusammenfassen:

Voraussetzung für eine Behandlung mit Akupunktur ist die Einhaltung der Hygiene- und aller Sicherheitsstandards, deshalb ist bei Erkrankten eine Selbstbehandlung sinnvoll und sollte vorgeschlagen werden (Akupressur, Moxibustion, Qigong/ Taiji).

Wirkmodelle für die Wirkung der Akupunktur bei derartigen Krankheiten sind Metaanalysen, die eine Wirkung zum Beispiel des Punktes S36/Ma36 („Dritter Weiler am Fuß“, zusanli) bei inflammatorischen Prozessen und im Tierversuch sogar eine Reduktion von Organschäden nach Sepsis zeigen. Mögliche Wirkme- chanismen sind eine Verbesserung der Mikrozirkulation und eine Reduktion des oxidativen Stresses (Lai F, Ren Y, Lai C et al: (2020) Acupuncture at Zusanli (S36) for Experimental Sepsis: A systematic review. Evid Nas Comp Altern Med 2020:3620741).

Im vorliegenden Artikel versucht Prof. Liang Fanrong, einer der im Bereich der Aku-Moxa-Therapie renommiertesten und international bekannten Wissenschaftler Chinas, anhand historischer Aussagen, gesundheitspolitischer Erfahrungen und Leitlinien sowie pathophysiologischer Überlegungen Therapieempfehlungen zur Akupunktur, Moxibustion und Akupressur bei COVID-19 zu destillieren.

Verschiedene andere Arbeitsgruppen (National Health Commission der VR China, die World Federation of Acupuncture, das Tang-PoXue-Team des Xinniang-TCM Hospital in Hubei) haben ebenfalls Vorschläge dazu erarbeitet und klinische Erfahrungen gesammelt. Im Folgenden sei eine praktische Synopse daraus angeführt:

Steigerung des orthopathischen Qi (zhengqi) und des qi defensivum (Wehrenergie, weiqi) (Prophylaxe COVID-19):

Täglich bzw. jeden zweiten Tag Behandlung mit Akupunktur oder Moxibustion oder Akupressur der Foramina Rs8/Ren8 („Mitte des Nabels“, shenque), S36/Ma36 („Dritter
Weiler am Fuß“, zusanli), Rs4/Ren4 („Das erste der Passtore“, guanyuan), Rg14/Du14 („Punkt aller Strapazen“, dazhui), Rs12/Ren12 („Sammlungspunkt des ,Magen'-Funktionskreises", zhongwan), V13/Bl13 („Einflusspunkt des ,Lungen'-Funktionskreises", feishu).

Behandlung leichter bis mittelschwerer Fälle:

Täglich bzw. jeden zweiten Tag Behandlung mit Akupunktur oder Moxibustion oder Akupressur der Foramina V12/Bl12 („Pforte der Winde“, fengmen), V13/Bl13 („Einflusspunkt des ,Lungen'-Funktionskreises", feishu), V20/Bl20 („Einflusspunkt des, Mitten'Funktionskreises“, pishu), IC4/Di4 („Vereinte Täler“, hegu), IC11/Di11 („Gekrümmter Teich“, quchi), P6/Lu6 („Die Tiefe des Lochs“, kongzui), P10/ Lu10 („Fischbauchgrenze“, yuji), S36/ Ma36 („Dritter Weiler am Fuß“, zusanli), L6/Mi6 („Die Verbindung der drei Yin“, sanyinjiao), Rg14/Du14 („Punkt aller Strapazen“, dazhui).

\section{Rekonvaleszenzphase:}

Täglich bzw. jeden zweiten Tag Behandlung mit Akupunktur oder Moxibustion oder Akupressur der Foramina S36/Ma36 („Dritter Weiler am Fuß“, zusanli), Rs4/Ren4 („Das erste der Passtore“, guanyuan), Rg14/Du14 („Punkt aller Strapazen“, dazhui), Rs12/ Ren12 („Sammlungspunkt des ,Magen-Funktionskreises", zhongwan), V13/Bl13 („Einflusspunkt des ,Lungen'-Funktionskreises“',feishu). 


\section{TCM-Quiz 91}

\section{von Rainer Nögel}

Frage 1: Zeichen einer algor-Heteropathie („Kälte“-Schrägläufigkeit, hanxie) sind
a) p. fixus („haftender“ Puls, laomai)
b) p. tardus (,verlangsamter“ Puls, chimai)
c) wandernde Schmerzen
d) Schweißlosigkeit
e) verlangsamte Sprache

Frage 2: Der Wandlungsphase Erde
a) ist keine Jahreszeit zugeordnet
b) entsprechen die jeweils letzten 18 Tage einer Jahreszeit
c) entspricht der Spätsommer
d) entspricht das yin maior (Großes Yin, taiyin)

Frage 3: Hohes Fieber, starkes Schwitzen, großer Durst und p. magnus („großer“ Puls, damai) sprechen für
a) Affektion des splendor yang (Überstrahlung des Yang, yangming)
b) depletio qi defensivum (energetische Schwäche der Wehrenergie, weiqi $x u$ )
c) Affektion der Qi-Ebene
d) calor intimae o. pulmonalis (innere „Hitze“ des Fk „Lunge“, fei li re)
e) Affektion der Ebene des qi constructivum (Bauenergie, yingqi)

Frage 4: Zur Behandlung der obigen Störung wären am ehesten geeignet

a) S40/Ma40 („Üppige Füll““, fenglong), Pc6/Pe6 („Inneres Passtor“, neiguan), V12/Bl12 („Pforte der Winde“, fengmen), P7/Lu7 („Reihe von Lücken“, lieque)

b) R3/Ni3 („Mächtiger Wasserlauf“, taixi), P9/Lu9 („Großer Wasserschlund“, taiyuan), S36/Ma36 („Dritter Weiler am Fuß“, zusanli), V13/Bl13 („Einflusspunkt des ,Lungen‘Funktionskreises", feishu)

c) IC4/Di4 („Vereinte Täler“, hegu), P9/Lu9 („Großer Wasserschlund“, taiyuan), S40/Ma40 („Üppige Fülle“, fenglong), T6/3E6 („Der fliegende Tiger“, feihu, bzw. „Seitlicher Abzugsgraben“, zhigou)

d) IC11/Di11 („Gekrümmter Teich“, quchi), S45/Ma45 („Die unterdrückte Heiterkeit“, lidui), P11/Lu11 („Junges Shang“, shaoshang), V13/Bl13 („Einflusspunkt des ,Lungen'-Funktionskreises“, feishu)

Frage 5: Welche Rezeptur würde sich zur Behandlung der obigen Störung anbieten?
a) „Dekokt mit Cinnamomum“ (Guizhi tang)
b) „Dekokt mit Ephedra, Prunus armeniaca, Gypsum und Glycyrrhiza“ (Maxing shigan tang)
c) „Dekokt des weißen Tigers“ (Baihu tang)
d) „Pulver zur Zerstreuung des Weißen“ (Xiebai san)
e) „Pulver mit Lonicera und Forsythia“ (Yinqiao san)

Frage 6: Die mm. animantia xue (das Xue bewegende Arzneimittel“, huoxue yao) werden zur Wirkungsverstärkung

a) häufig durch Rösten mit Honig (mizhi) aufbereitet

b) häufig durch Rösten mit Essig (cuzhi) aufbereitet

c) häufig durch Rösten mit Wein (cuzhi) aufbereitet

d) häufig durch Rösten mit Weizenkleie (fuzhao) aufbereitet

\section{Auflösung der Fragen auf Seite 94}

